\title{
ROYAL PHOTOGRAPHIC SOCIETY'S EXHIBITION
}

$\mathrm{T}$ HE ninety-seventh annual exhibition of the Royal Photographic Society was opened in London on September 12 for one month and will also be shown in the City Art Gallery of Leeds during October 25-November 23 and in the Art Gallery of Bristol during December 6-31. The exhibition is divided into seven sections: pictorial, scientific, medical, nature, stereoscopic, record and kinematic photography, with a total of 849 exhibits.

Within the Scientific Section, exhibits confined to photographic applications of physics and chemistry are small in number, though not in quality, while entries of biological interest are reasonably well represented. The Aerodynamics Division of the National Physical Laboratory is showing three prints consisting of Schlieren patterns of the air disturbances emanating from aerofoils mounted in an air current of Mach number 1.6. Two of these prints are in colour and show the various regions of compression and rarefaction by making use of the familiar technique which employs polarized light for the exposure. P. H. Harris shows an electron micrograph of a sheet of etched aluminium foil in which the otching had eaten away the metal, apparently by boring it out in the form of minute tunnels. A. V. C. Cross is exhibiting a series of photomicrographs in which are shown the gradual erosion of a film of rosin naphtha varnish by sea water. A number of aerial survey photographs by Hunting Aerosurveys, Ltd., principally concerned with the site for a dam in Iraq, are of considerable geological interest. In addition, an aerial photograph of the ruined Palace of Persian Kings of Persepolis gives a strong impression of the beauty and splendour that such a building must have possessed in ages past. One of the biological exhibits by H. J. Howard consists of a series of four photomicrographs of progressively increasing magnification from $\times 10$ to $\times 350$ of the apparently simple bird's feather. First the shafts of the feather, then the barbs and finally the barbules are progressively brought into prominence, the fibres of the last interlocking almost at right angles to produce an intricate and effective protection. T'he selective absorption of silver on the gills of a freshwater crab is shown in an exhibit by S. F. Bush, yet the particulars of what is clearly a process of considerable interest to the chemist are left unexplained. A good example of phase-contrast microscopy by B. A. Jarrett consists of the micro-organism Stentor roselii, in which it can be seen how movements of the cilia enable the cell to swim. An excellent series of colour transparencies by G. H. McLean shows the effects of five different diseases on the leaves of the sugar-beet plant.

In the Medical Section, many of the exhibits hold more than a morbid interest to the lay spectator, because they adequately describe some cycle or technique rather than illustrate some more-or-less distasteful specimen under an obscure Latin title. For example, a virus culture technique shown by the Institute of Ophthalmology, London, is most clearly demonstrated in a series of pictures in which, starting with an eleven-day old fertile incubated egg, part of the shell has been cut away and the egg inoculated. Incubation was continued for about three days, after which the membrane was covered with virus lesions.
Perhaps one of the most noteworthy exhibits is from the Hospital for Sick Children, Great Ormond Street, London, in which photographs of a patient taken as a boy and also as a man forty-seven years later show what was probably the first successful surgical removal of a brain tumour with survival over this period. From the same hospital there is also shown, in another exhibit, the system employed for the identification and recording of the particulars of each patient. The technique employed in testing such antibiotics as aureomycin, chloromycetin, penicillin, streptomycin and terramycin is shown by $\mathbf{S}$. E. Veronique. Small paper disks impregnated with the antibiotic are placed on the surface of agar upon which Staphylococcus is seeded, and the clear area around the paper disks in which growth of the organism is inhibited is a measure of the effectiveness of the antibiotic. The Manchester Royal Infirmary is showing how enlargement of a radiograph can prove to be worth while, and illustrates this with $\times 10$ enlargements of pathological bone specimens originally exposed on ordinary commercial fine-grain film. Further exhibits by the Institute of Ophthalmology demonstrate techniques and apparatus for retinography and keratography together with colour prints of the human retina. A series of mounts from Guy's Hospital, London, are interesting in that they record limiting movements of the hand, foot or eye either by taking more than one exposure on the same negative or else registering the pictures showing extremes of movement and making tracings from the separate pictures. Photographs of some of the appliances which come to the aid of a hand deprived of normal movement are also shown by K. G. Moreman.

There are numerous excellent exhibits in the Nature and Record Sections of the Exhibition which make it difficult to review with justice; but perhaps the entries on the life-histories of marsh fritillary, gipsy moth, vapourer moth and emperor moth by S. Beaufoy and J. D. C. Boyes deserve special mention.

\section{MODERN METHODS IN THE TREATMENT OF TUBERCULOSIS}

7 HE figures for tuberculosis mortality (England and Wales) since the end of the Second World War show a marked decline. In 1946 the deaths from respiratory tuberculosis were 19,365; in 1950 they were 14,079. In 1946 the deaths from other forms of tuberculosis (bone and joint disease, meningitis, glandular disease) were 3,485 ; in 1950 they were 1,890 .

The mortality from tuberculosis in England and Wales, moreover (with the exception of temporary set-backs during two World Wars), has been steadily declining since 1912, when the national scheme for combating tuberculosis, now merged in the National Health Service, was established. Even allowing for the potential influence of a hypothetical epidemiological cycle, it is not unreasonable to assume that increased knowledge and wisely directed administration have contributed to this decline. 
There are certain methods of prevention, diagnosis and treatment which are powerful allies in the anti-tuberculosis campaign. These are as follows.

(1) Research. There is close association of research work in tuberculosis with practical application by clinicians. Important work in this field is done by tuberculosis committees of tho Medical Research Council, the Ministry of Health, the Prophit Trust of the Royal College of Physicians, the Papworth Research Institute, the Tuberculosis Association and other research bodies. Studies are being made on immunization with B.C.G.

(2) Pasteurization of milk. It is estimated that 30 per cent of non-pulmonary tuberculosis is due to infection by bovine strains of the tubercle bacillus, almost always conveyed by milk. In England some 80 per cent of liquid milk is heat-treated, and if only the remaining 20 per cent were so treated a further large reduction in these deaths might well follow.

(3) $X$-rays. In the past twenty years the improvement in X-ray technique has helped the diagnosis of pulmonary tuberculosis and the control of collapse therapy. The value of tomography was established by the work of MeDougall and Turning in Great Britain. Lastly came mass radiography, which has developed much of late years for the diagnosis of the early case of pulmonary tuberculosis.

(4) Surgical treatment. The extended use of artificial pneumothorax from 1920 onwards marked a great advance. It may be associated, however, with certain severe complications, such as air embolism, spontaneous pneumothorax, empyema and bronchopleural fistula. Difficulties also arise in the termination of an artificial pneumothorax, for the lung will not always re-expand. The type of case, a limited lesion, which did well with this treatment is often treated by chemotherapy to-day. Artificial pneumothorax is still of value in appropriate cases, although some practitioners, especially in Holland, have abandoned it in favour of other surgical measures. Pneumoperitoneum is still employed, both as a useful accessory to chemotherapy and as a temporary procedure to prepare a patient for operation; but phrenic crush, which partly paralyses the diaphragm, often permanently limits respiratory function and is seldom advised. $R$. Laird is of opinion that thoracoplasty (usually done in two stages) has still a definite place in the treatment of pulmonary tuberculosis. In the six years 1944-50, of 580 patients treated in this way there was a mortality-rate of less than 1 per cent per operation, and 72 per cent of these patients were sputumnegative during the two-months before their discharge. Various extrapleural and extrafascial operations may be grouped with thoracoplasties. A one-stage operation followed by no deformity is attractive, but the fate of the dead space and its plastic packing has yet to be determined. Cavity drainage is not often done in Great Britain. Excision of diseased lung formerly had a high mortality from the risk of spread of tuberculous infection. Improvement in operative technique and the use of antibiotics, notably streptomycin, have made pneumonectomy, lobectomy, segmental resection and wedge excision safer and of benefit to many patients unsuitable for collapse therapy. In Laird's series, resection of lung gave a sputum-conversion rate of more than 90 per cent, and the mortality among 132 patients so treated was 4.5 per cent. Despite the increased risk, excision in the United States has largely replaced thoracoplasty, and it is an operation which has now a definite place in treatment.

(5) Chemotherapy. The clinical application of antibiotics, streptomycin and $p$-aminosalicylic acid is being actively pursued with encouraging results. Many patients with pulmonary tuberculosis are initially thus treated at home, and, consequently, a higher proportion of those admitted to sanatoria for active treatment have become sputum-negative. This favourably influences medical and surgical treatment alike in sanatoria.

Prevention and treatment must go hand-in-hand in the attack on tuberculosis. As infected cases in the community become fewer, so will the prevalence and mortality of the disease further decline. The new methods of treatment here discussed are highly encouraging; but the older and simpler methods of prevention, notification, contact examination, environmental hygiene and good nutrition must be associated with them.

Arthur MacNaLty

\section{REFERENCES}

MacNalty, A. S., "The Control of Pulmonary Tuberculosis in England", Brit. J. Tuberculosis, 45, 90 (1951).

Sellors, T. H. "The Evolution of Thoracic Surgery", Brit. J. Tuberculosis 45, 97 (1951).

Simmonds, F. A. H., Laird, R., and Macdonald, N., "Recent Trends in the Treatment of Pulmonary Tuberculosis", Lancet, ii, 347 (1952).

\section{CARNEGIE UNITED KINGDOM TRUST \\ REPORT FOR 1951}

$T$ HE thirty-eighth annual report of the Carnegie United Kingdom Trust, covering the year 1951*, refers to the impeding of the village-hall building programme by the general restrictions on capital expenditure : work proceeded on twenty-one schemes throughout Great Britain, and grant instalments amounting to $£ 11,679$ were paid; a sum of $£ 30,000$ remains available for schemes approved before the end of 1947 but still awaiting a starting date.

Sums of $£ 15,000$ were allocated to the Family Welfare Association and to Family Service Units for the quinquennium. The former is conducting an experiment in the approach to personnel problems from the point of view of community relationships and also another experiment involving the employment of a specially trained worker to deal with problem families as part of the case-load at one of the Association's centres. The Family Service Units undertake practical work in the homes of problem families and, as a corollary, practical research in close association with the field-work of the separate units now established in some of the larger cities. A grant of $£ 6,000$ to Stanley House, a community centre in Liverpool for coloured people and their friends, is for a dovelopment plan involving structural alterations and provision of additional facilities to make the centre a house of friendship for everyone, irrespective of colour, class or creed. Tho Joint Committee on Museums Policy is surveying several provincial museums which have been selected as potential centres for installing experimental demonstration galleries embodying the most modern ideas of the technique of presentation.

* Carnegie United Kingdom Trust. Thirty-eighth Annual Report 1951. Pp. viii +48. (From the Trust, Dunfermline, Hife, 1952.) 